\title{
MENSURAÇÃO E AVALIAÇÃO DE IMPACTO SOCIAL DE EMPREENDIMENTOS SOCIAIS
}

MEASUREMENT AND EVALUATION OF SOCIAL IMPACT OF SOCIAL ENTERPRISES

Recebido em 16.08.2020 Aprovado em 22.09.2020

Avaliado pelo sistema double blind review

DOI: https://doi.org/10.12712/rpca.v14i3.44590

\section{Elisa Pereira Murad}

murad.elisa@gmail.com

Programa de Pós-Graduação em Administração/Universidade Federal de Lavras - Lavras/Minas Gerais, Brasil https://orcid.org/0000-0003-1266-9433

\section{Mônica Carvalho Alves Cappelle}

edmo@dae.ufla.br

Programa de Pós-Graduação em Administração/Universidade Federal de Lavras - Lavras/Minas Gerais, Brasil https://orcid.org/0000-0002-0095-3405

\section{Daniela Meirelles Andrade}

daniela.andrade@ufla.br

Programa de Pós-Graduação em Administração/Universidade Federal de Lavras - Lavras/Minas Gerais, Brasil https://orcid.org/0000-0001-7893-4629

\section{Resumo}

Este trabalho compreende uma revisão integrativa que tem como objetivo identificar metodologias e ferramentas utilizadas para acompanhar, mensurar e avaliar o impacto social de empreendimentos sociais e discutir suas características e potenciais de contribuição. A revisão foi realizada a partir da literatura nacional e internacional, e possibilitou constatar que na maioria dos estudos o foco encontra-se na identificação de indicadores de resultado. Conclui-se que é necessário discutir a mensuração de impacto em uma perspectiva de processo e de envolvimento das partes interessadas. Para tanto, deve-se ter o conhecimento do contexto e das necessidades presentes, tendo como foco a comunidade e a sua participação.

Palavras-chave: Empreendedorismo social. Mensuração de impacto social. Avaliação de impacto social. Indicadores de resultado.

\begin{abstract}
This paper comprises an integrative review that aims to identify methodologies and tools used to follow, measure, and evaluate the social impact of social enterprises and discuss their characteristics and contribution potentials. The review was carried out from the national and international literature and made it possible to note that in most studies the focus is on the identification of result indicators. Concludes that it is necessary to discuss impact measurement from a process and stakeholder involvement perspective. To do so, one must have knowledge of the context and present needs, focusing on the community and its participation.
\end{abstract}

Keywords: Social entrepreneurship. Social impact measurement. Social impact assessment. Outcome indicators. 


\section{Introdução}

Diante dos problemas sociais existentes que afetam o desenvolvimento da sociedade, surgem empreendimentos direcionados a essas questões, tendo como objetivo principal a geração de valor social (Austin, Stevenson \& Wei-Skillern, 2006; Corner \& Ho, 2010; Dacin, Dacin \& Tracey, 2011; Defourny \& Nyssens, 2010), que seriam os benefícios oferecidos à população, incluindo saúde, educação, qualidade de vida e melhorias ecológicas (Clark \& Brennan, 2016). Estes se apresentam como uma forma de gerar mudança social (Mair \& Marti, 2006), ao atender as necessidades básicas da população, as quais não são atendidas pelo mercado ou instituições públicas (Santos, 2012; Seelos \& Mair, 2005).

Neste contexto, o foco do empreendimento é a geração de valor social enquanto o valor econômico é condição necessária para garantir a viabilidade financeira do mesmo (Mair \& Marti, 2006; Saebi, Foss \& Linder, 2018), percebida como subproduto que permite que a organização tenha sustentabilidade e autossuficiência, não sendo dependente de doações de recursos (Seelos \& Mair, 2005).

Essa breve descrição refere-se ao Empreendedorismo Social (ES), o qual proporciona impacto positivo na sociedade, aproveitando oportunidades, detectando falhas de mercado, e criando soluções inovadoras para as demandas sociais (Dees, 1998). Neste artigo, a definição de ES está fundamentada em Oliveira (2004), o qual define ES como ações inovadoras direcionadas para questões sociais, que partem da observação de um contexto e de um problema local, de forma que se busca elaborar uma alternativa de enfrentamento. A iniciativa deve ser inovadora, realizável, autossustentável, envolver várias pessoas e segmentos da sociedade (principalmente a população atendida), provocar impacto social e fazer com que os resultados possam ser avaliados.

Quanto às transformações e impactos sociais gerados, é importante destacar que o termo aqui empregado se refere às mudanças que ocorrem na sociedade a partir do momento que se desenvolve um projeto (Brandão et al, 2014). Para o relatório The Code of Good Impact Practice (2013), impactos sociais são efeitos de longo prazo gerados pelo trabalho de uma organização, podendo ser referido como a diferença que se realiza em um determinado contexto. A questão de impacto social de um projeto é vista como um ponto que merece atenção, pois o foco de empreendimentos sociais é o retorno social que será obtido (Ashoka \& McKinsey, 2001).

Nesse contexto, desenvolver mensuração de impacto social torna-se imperativo, pois organizações sociais estão sendo, de certa forma, pressionadas a explicitar o seu valor social, e assim, para evidenciar os resultados é necessário mensurar (Clifford, Markey \& Malpani, 2013). Mair e Marti (2006) discutem que avaliar o impacto social é um dos maiores desafios para profissionais e pesquisadores do campo, onde o problema pode não ser a mensuração em si, mas como as medidas podem ser usadas para "quantificar" e qualificar o impacto, transformando em dados os impactos socioeconômicos, ambientais, efeitos mentais e sociais obtidos.

Embora seja difícil avaliar o desempenho de um empreendimento social, existem mecanismos que ajudam nessa questão (Certo \& Miller, 2008), por meio de ferramentas e metodologias de mensuração e avaliação de impacto social, e diante disso, pesquisas sobre relatórios de impacto social precisam ser desenvolvidas com maior profundidade (Nicholls, 2009). Portanto, tendo em vista a importância dessa questão, o presente trabalho busca responder à seguinte pergunta: Quais são e como se caracterizam as metodologias e ferramentas de mensuração e avaliação de impacto social de empreendimentos sociais, abordadas na literatura nacional e internacional?

Para responder a essa pergunta, realizou-se uma revisão integrativa sobre o tema de mensuração e avaliação de impacto social, a partir de periódicos nacionais e internacionais com o objetivo de identificar metodologias e ferramentas utilizadas para acompanhar, mensurar e avaliar o impacto social de ES e discutir suas características e potenciais de contribuição para esses empreendimentos. 
Esta pesquisa justifica-se por almejar contribuir com os estudos científicos ao apresentar e discutir ferramentas e metodologias de mensuração de impacto social, com ênfase no processo e na participação das partes interessadas, que são questões que precisam ganhar destaque, tendo em vista as lacunas percebidas. Assim, é importante, como defendido por Mair e Marti (2006), estabelecer legitimidade do conceito como área de investigação acadêmica. Não apenas na área acadêmica, mas empiricamente vê-se a necessidade de estudos para apoiar as organizações sociais que estão sendo, de certa forma, pressionadas a tornarem evidente o valor social que tem proporcionado. Com isso, é possível gerar mecanismos para melhorar o desempenho das ações, atrair e adquirir recursos e construir legitimidade organizacional (Barman, 2007; Nicholls, 2009), os quais possibilitarão resultados comprometidos com o objetivo dessas organizações.

Este trabalho apresenta primeiro a introdução, a qual contempla o problema e objetivo de pesquisa. Em um segundo momento, tem-se o referencial teórico distribuído em duas seções: a primeira aborda o tema de empreendedorismo social e a segunda apresenta o conceito de avaliação e mensuração de impacto social. Em seguida apresenta-se a metodologia adotada, e após este tópico, tem-se a apresentação e discussão dos resultados. Por fim, são apresentadas as considerações finais e as referências bibliográficas do trabalho.

\section{Empreendedorismo social}

O conceito de empreendedorismo social (ES) emergiu na década de 90 e desde então passou a receber atenção pela academia, assim como também começou a ser tratado como um componente-chave da agenda de desenvolvimento do século XXI (Abdo \& Paris, 2017). O termo está ligado às iniciativas que tem como objetivo principal a geração de valor social (Austin, Stevenson \& Wei-Skillern, 2006; Corner \& Ho, 2010; Dacin, Dacin \& Tracey, 2011; Defourny \& Nyssens, 2010), buscando gerar mudança social (Mair \& Marti, 2006), ao atender as necessidades básicas da população, as quais não são atendidas pelo mercado ou instituições públicas (Santos, 2012; Seelos \& Mair, 2005).

Este é um conceito contestado, de forma que existem muitas definições, mas não existe um quadro conceitual unificado (Choi \& Majumdar, 2014). Bacq e Janssen (2013) identificaram três principais escolas de pensamento sobre a temática, duas delas originadas dos EUA: The Social Innovation School enfatiza as características do indivíduo empreendedor social, focada na perspectiva do indivíduo; a segunda, The Social Enterprise School, afirma que o empreendimento sobrevive realizando atividades que geram lucros para dar sustento à geração de valor; a terceira está relacionada com a abordagem europeia, mais ligada às empresas sociais, e nessa perspectiva o foco está voltado em modelos coletivos, ao invés do indivíduo empreendedor.

A missão social é tratada como objetivo principal do empreendimento, permeando tanto as abordagens norte-americanas quanto a europeia, e as diferenças existentes entre os modelos está ligada a fatores contextuais, pois mesmo nos EUA existem diferentes noções (Bacq \& Janssen, 2013). Assim, as concepções de ES estão enraizadas nos contextos sociais, econômicos, políticos e culturais onde as organizações emergem, portanto, para a compreensão do fenômeno, devem-se levar em conta as especificidades dos locais que moldam essas iniciativas de diferentes formas (Defourny \& Nyssens, 2010). Diante disso, é importante entender que o desenvolvimento de um empreendimento não pode ser apenas analisado na perspectiva de exportação de abordagens dos Estados Unidos e da Europa, devendo deslocar essas discussões que geralmente são direcionadas nessas duas localidades (Defourny \& Nyssens, 2010).

Ao tratar de ES, faz-se também necessário destacar algumas diferenças em relação a determinados termos. Este tipo de empreendimento se difere de filantropia e caridade, bem como de responsabilidade social empresarial, sendo que nesta última são encontradas ações que estão de acordo com a missão e atividade da empresa, acima das necessidades da comunidade (Oliveira, 2004). O ES também se difere do empreendedorismo tradicional, o qual possui como prioridade gerar valor econômico, ou seja, lucrar, 
enquanto o social tem como foco principal gerar impacto social, procurando soluções para os problemas que afetam a sociedade (Azevedo, 2015; Barki et al., 2015; Oliveira, 2004; Saebi, Foss \& Linder, 2018; Yunus, Moingeon \& Lehmann-Ortega, 2010).

Para Austin, Stevenson e Wei-Skillern (2006) o termo, embora muito associado às iniciativas realizadas por organizações sem fins lucrativos, também permite um entendimento que envolve empresas e organizações públicas. Nesse contexto, o surgimento de oportunidades é resultante das redes de atores interconectados que combinam seus recursos e habilidades para alcançar uma ação social efetiva (Corner \& Ho, 2010).

O foco principal do empreendimento se encontra na geração de valor social enquanto o valor econômico é visto como condição necessária para garantir a viabilidade financeira (Mair \& Marti, 2006). Para Mair e Marti (2006) o empreendedorismo social não pode ser entendido exclusivamente pelo sentido econômico, e deve ser examinado à luz do contexto social e ambiental. Para ser efetivo é preciso envolver a comunidade, compreender a cultura local e o contexto, para assim gerar soluções para os mesmos (Azevedo, 2015).

Diante do exposto, é necessário entender que não se deve querer sanar apenas problemas imediatos, mas sim modificar o sistema com medidas que realizam importantes transformações, trazendo melhorias à determinada comunidade. Essas transformações incluem a mobilização de recursos e os impactos, que trazem mudanças ao contexto social em que emerge o problema (Alvord, Brown \& Letts, 2004).

Para acompanhar e verificar se essas mudanças estão de fato ocorrendo, é necessário haver avaliação de impacto, questão esta que é tratada como um dos maiores desafios para profissionais e pesquisadores do campo (Mair \& Marti, 2006). Os empreendedores usam os relatórios de impacto social na perspectiva de estratégia para melhorar o desempenho de suas ações, adquirir recursos e construir legitimidade organizacional, e assim, deve-se buscar não apenas melhorar o desempenho interno, mas também manter a responsabilização pelo externo, diante das organizações sociais, comerciais e do setor público (Nicholls, 2009).

Nesse cenário, o argumentar de que não há mecanismos para mensurar o resultado social é visto como um discurso que reforça a incapacidade de gestão da organização (Azevedo, 2015), tendo em vista que existem materiais, ferramentas e metodologias para apoiar as organizações nessas atividades, questão essa que será tratada no próximo tópico.

\section{Mensuração e avaliação de impacto social}

Embora seja difícil avaliar o desempenho de um empreendimento social existem mecanismos que auxiliam nessa questão (Certo \& Miller, 2008), e, como apresentado por Mair e Marti (2006), é importante estabelecer legitimidade do conceito como área de investigação acadêmica. Assim, os empreendimentos sociais, com fins lucrativos ou não, devem identificar métricas de impacto social baseadas em sua missão e no impacto realizado (Neck, Brush \& Allen, 2009).

Nota-se que as organizações sociais estão sendo, de certa forma, pressionadas a tornar evidente o seu valor social (Clifford, Markey \& Malpani, 2013). Essa pressão ocorre tendo em vista que é necessária a mensuração das atividades da organização para poder demonstrar sua competência, adquirir legitimidade e obter fundos (Barman, 2007). Barki et al. (2012) apresentam que a capacidade de uma organização mensurar seus impactos gera para ela um diferencial, pois é um atrativo para investimentos de impacto. Para esses autores, a mensuração de impacto social não é algo simples, pois deve permitir analisar impactos de longo prazo e não apenas os imediatos.

Não há uma visão consensual e abrangente sobre como a atividade de mensuração de resultados sociais deve ser realizada, ou seja, quais os métodos que devem ser utilizados (Azevedo, 2015). Notam-se 
pesquisas surgindo nos últimos anos sobre mensuração, contudo esse campo ainda não está bem estruturado (So \& Staskevicius, 2015).

Ogain (2015) apresenta algumas questões que facilitam o desenvolvimento de estratégias de impacto para o empreendimento social, são elas: i) identificar qual problema social a organização visa atender, quais são as causas raízes e as formas de combater; ii) identificar a inovação e mostrar evidências de que a mesma trará impacto; iii) mapear a teoria da mudança para entender o impacto que irá gerar; iv) traçar estratégias para que o empreendimento tenha escala e sustentabilidade; v) pensar em métricas e tipos de avaliação que terá que criar.

Carol Weiss propôs uma alternativa de avaliação, conhecida por "Theories of Change", para auxiliar as iniciativas, a qual virou referência. De acordo com Weiss (1995) essas iniciativas têm o potencial não apenas para fazer o bem, mas para entender como, quando e porque o bem está sendo realizado, devendo assim compreender que com os processos de mudança será possível obter sucessos e trazer benefícios para o público-alvo.

Nota-se que há casos de avaliações que apresentam as atividades e os resultados alcançados, mas não especificam os mecanismos causais, ou seja, a relação entre os elementos que levarão à mudança social pretendida (Rogers \& Weiss, 2007). Nesta situação apresentada, foca-se em uma estratégia de mensuração que trata o processo de forma linear, representado por um modelo lógico, onde estão indicados os elementos envolvidos para gerar o impacto, mas não há discussão sobre a relação entre eles e as premissas envolvidas (Roger \& Weiss, 2007). Diante disso, faz-se necessário amadurecer a teoria para o impacto ser gerado.

Outra questão que ocorre, é quando a lógica por trás do projeto é baseada em suposições ou raciocínio lógico de quem pratica, sem haver imersão em um contexto específico. Portanto, neste caso, ao ser elaborada a metodologia de mensuração, deve-se apresentar uma série de micro etapas (recursos, atividades, produtos e serviços, resultados e impacto), atribuindo relações causais entre os elementos presentes nas etapas, entendendo o processo desde os insumos até o resultado, de acordo com os propósitos da intervenção (Rogers \& Weiss, 2007).

Para Rogers e Weiss (2007), uma avaliação tem que envolver um trabalho que possibilite definir e medir processos psicossociais, fisiológicos, econômicos, sociológicos, organizacionais, dentre outros, formando projetos que tem bases em premissas melhor concebidas. Para tanto, os avaliadores precisam ter conhecimento a respeito de questões sociológicas em busca de pistas para formulações válidas, desenvolvendo assim intervenções sociais que resultarão em um impacto positivo.

Para avaliar o impacto é necessário se pautar em determinados indicadores, os quais contêm informações que apresentam as mudanças derivadas de uma intervenção, e assim são utilizadas para monitoramento e avaliação (Church \& Rogers, 2006). Parkinson e Wadia (2008) apresentam que com uso de indicadores é possível verificar se a organização está alcançando seus objetivos e metas, sendo possível assessorar o progresso do projeto. Indicadores são como fator de motivação, ao ser possível reconhecer a mudança que está realizando. Eles possibilitam: i) apresentar se o objetivo foi alcançado; ii) analisar como os usuários tem se beneficiado com a intervenção; iii) identificar lacunas; iv) realizar melhorias.

Na prática, nota-se o reconhecimento da importância da mensuração de impacto social, mas também se encontram desafios quanto ao desenvolvimento de indicadores para medir os resultados obtidos pela organização (Tyszler, 2007), e a formalização de um instrumento para medir impacto que seja conhecido e compreendido pelos envolvidos nos projetos (Kuyumjian, Souza \& Sant'anna, 2014).

Portanto, tendo em vista a importância da mensuração de impacto social, a dificuldade de ser realizada na prática e a escassez de estudos voltados a essa temática, é possível notar a necessidade de discutir esse 
conceito para que se desenvolvam estratégias adaptadas à realidade do contexto, de forma que atendam as demandas dos empreendimentos e da sociedade.

\section{Procedimentos metodológicos}

Este trabalho contempla uma revisão integrativa da literatura sobre o tema de mensuração e avaliação de impacto social. Botelho, Cunha e Macedo (2011) apresentam que o processo de revisão de literatura possibilita a compreensão sobre o conhecimento gerado e oferece base para a construção do conhecimento científico, dando possibilidade ao surgimento de novas teorias. Para esses autores, a revisão integrativa é um método que permite o desenvolvimento de uma revisão de literatura e possibilita a síntese e análise do conhecimento científico produzido a respeito de um tema investigado. Favorece assim, a aproximação do pesquisador a uma problemática e a visualização de possíveis oportunidades de pesquisa.

Para Botelho, Cunha e Macedo (2011), a realização de uma pesquisa integrativa envolve os seguintes passos: i) etapa da identificação do tema e seleção da questão de pesquisa; ii) etapa de estabelecimento de critérios de inclusão e exclusão; iii) etapa de identificação dos estudos pré-selecionados e selecionados; iv) etapa de categorização dos estudos selecionados; v) etapa de análise e interpretação dos resultados; vi) etapa de apresentação da revisão/síntese do conhecimento.

Portanto, partindo da problemática de pesquisa, como etapa inicial foram realizadas buscas de artigos nas bases Scopus, SciELO e Google Scholar, sem delimitação de período, envolvendo pesquisa em título, resumo e palavras-chaves, selecionando documentos do tipo "Artigo", publicados em revistas científicas, em todas as áreas. Para a busca de artigos nacionais, utilizaram-se as bases SciELO e Google Scholar, contudo ocorreu uma flexibilização quanto aos termos, devido à dificuldade de encontrar artigos envolvendo as temáticas de empreendedorismo social e mensuração de impacto social. A escolha das bases se deu por abrangerem uma diversidade de produções bibliográficas, mostrando-se úteis nas buscas de artigos para a temática estudada.

Os critérios utilizados para seleção dos artigos foram: i) artigos que tratam a temática de empreendedorismo social; ii) artigos que abordam mensuração de impacto social por empreendimentos sociais; iii) artigos que apresentam metodologias e/ou ferramentas de avaliação de impacto social. Quanto aos critérios de exclusão, foram adotados: i) artigos que tratam de empreendedorismo tradicional ou gestão pública; ii) mensuração em outras perspectivas, como por exemplo: impacto negativo, capital social, inovação etc; iii) artigos que não tratam de mensuração de impacto e/ou empreendedorismo social.

A pesquisa realizada na base Scopus utilizou os termos: i) "Measuring Social Impact" AND "Social Entrepreneurship"; ii) "Impact Measurement" AND "Social Entrepreneurship". Para busca na base SciELO, utilizaram-se os termos "Projetos Sociais" AND "Avaliação", e no Google Scholar: "Avaliação de Projetos Sociais" E "Empreendedorismo Social".

Os estudos identificados por meio da pesquisa nas bases foram: 62 pela Scopus (28 artigos na $1^{\text {a }}$ busca e 34 artigos na $2^{\text {a }}$ busca), 88 pela SciELO e 82 Google Scholar, totalizando 232 trabalhos. Desse total, 207 foram excluídos: 20 tratam de empreendedorismo tradicional, 15 de mensuração em outra perspectiva, 7 artigos duplicados, 9 não abordam mensuração de impacto, 123 não tratam de mensuração de impacto e de empreendedorismo social, e 33 não são artigos.

Portanto, a partir da leitura do resumo, das palavras-chave e dos títulos dos trabalhos foram préselecionados 25 artigos, a partir dos quais se realizou leitura integral dos textos e foram selecionados 12 para a pesquisa e excluídos 13. Desses 13, cinco não tratam de mensuração de impacto, seis não abordam metodologia de mensuração de impacto, e dois tratam do contexto público. 
Com a seleção dos artigos concluída, foi realizada uma análise dos dados coletados a partir da análise de conteúdo, que de acordo com Bardin (2009) envolve três momentos: primeiro, a descrição analítica dos dados coletados; segundo, contempla a sustentação da análise descritiva por meio do referencial teórico; terceiro, são desenvolvidas as categorias de análise que permitem a corroboração das perguntas levantadas na pesquisa.

No caso, as categorias de análise surgiram pela busca de resposta ao objetivo da pesquisa. Contemplaramse as seguintes categorias: i) metodologias e ferramentas de avaliação e mensuração de impacto social, e ii) foco dos artigos (processo, indicadores de resultado ou ambos). Portanto, houve o tratamento das categorias estabelecidas a partir da base conceitual sobre mensuração e avaliação de impacto e da leitura dos textos, alinhado com o objetivo proposto do trabalho.

\section{Apresentação e análise dos resultados}

\section{Mensuração e avaliação de impacto: a discussão central em artigos nacionais e internacionais}

A partir do objetivo de pesquisa, analisaram-se os textos selecionados considerando metodologias e/ou ferramentas tratadas sobre mensuração de impacto social. Com isso, possibilitou conhecer as formas utilizadas nos diferentes trabalhos para desenvolver uma estratégia de impacto e de mensuração, que esteja alinhada com os interesses da comunidade e com a missão social da organização.

Foram analisados 12 trabalhos, oito internacionais e quatro nacionais. A seguir, na Figura 1, estão apresentados os artigos que participam da análise, seguindo ordem alfabética, contemplando a indicação dos autores, título, ano, periódico, número de citações, tipo de estudo e base de dados.

\begin{tabular}{|c|c|c|c|c|c|c|}
\hline Autores & Título & Ano & Periódico & $\begin{array}{l}\mathbf{N}^{\circ} \text { de } \\
\text { Citações }\end{array}$ & $\begin{array}{l}\text { Tipo de } \\
\text { Estudo }\end{array}$ & $\begin{array}{c}\text { Base de } \\
\text { Dados }\end{array}$ \\
\hline \multicolumn{7}{|c|}{ Internacionais } \\
\hline $\begin{array}{l}\text { André, } \\
\text { Cho e } \\
\text { Laine }\end{array}$ & $\begin{array}{l}\text { Reference points for measuring } \\
\text { social performance: case study of } \\
\text { a social business venture }\end{array}$ & 2017 & $\begin{array}{c}\text { Journal of Business } \\
\text { Venturing }\end{array}$ & 5 & Empírico & Scopus \\
\hline $\begin{array}{l}\text { Clark e } \\
\text { Brennan }\end{array}$ & $\begin{array}{c}\text { Entrepreneurship with social } \\
\text { value: a conceptual model for } \\
\text { performance measurement }\end{array}$ & 2012 & $\begin{array}{c}\text { Academy of } \\
\text { Entrepreneurship } \\
\text { Journal }\end{array}$ & 10 & Teórico & Scopus \\
\hline $\begin{array}{l}\text { Clark e } \\
\text { Brennan }\end{array}$ & $\begin{array}{c}\text { Social entrepreneurship: a global } \\
\text { model for evaluating long-term } \\
\text { impact }\end{array}$ & 2016 & $\begin{array}{l}\text { International Journal } \\
\text { of Entrepreneurship }\end{array}$ & 1 & Empírico & Scopus \\
\hline $\begin{array}{l}\text { Grieco, } \\
\text { Michelini } \\
\text { e Lasevoli }\end{array}$ & $\begin{array}{l}\text { Measuring value creation in social } \\
\text { enterprises: a cluster analysis of } \\
\text { social impact assessment models }\end{array}$ & 2014 & $\begin{array}{c}\text { Nonprofit and } \\
\text { Voluntary Sector } \\
\text { Quarterly }\end{array}$ & 16 & Teórico & Scopus \\
\hline $\begin{array}{l}\text { Hadad e } \\
\text { Gauca }\end{array}$ & $\begin{array}{c}\text { Social impact measurement in } \\
\text { social entrepreneurial } \\
\text { organizations }\end{array}$ & 2014 & $\begin{array}{l}\text { Management \& } \\
\text { Marketing }\end{array}$ & 4 & Teórico & Scopus \\
\hline Manetti & $\begin{array}{c}\text { The role of blended value } \\
\text { accounting in the evaluation of } \\
\text { socio-economic impact of social } \\
\text { enterprises }\end{array}$ & 2012 & Voluntas & 20 & Teórico & Scopus \\
\hline $\begin{array}{l}\text { Moody, } \\
\text { Littlepage } \\
\text { e Paydar }\end{array}$ & $\begin{array}{l}\text { Measuring Social Return on } \\
\text { Investment: lessons from } \\
\text { organizational implementation of } \\
\text { SROI in the Netherlands and } \\
\text { The United States }\end{array}$ & 2015 & $\begin{array}{l}\text { Nonprofit } \\
\text { Management \& } \\
\text { Leadership }\end{array}$ & 6 & Empírico & Scopus \\
\hline $\begin{array}{l}\text { Spencer, } \\
\text { Brueckner } \\
\text {, Wise e } \\
\text { Marika }\end{array}$ & $\begin{array}{l}\text { Australian indigenous social } \\
\text { enterprise: measuring } \\
\text { performance }\end{array}$ & 2016 & $\begin{array}{l}\text { Journal of } \\
\text { Enterprising } \\
\text { Communities }\end{array}$ & 4 & Empírico & Scopus \\
\hline
\end{tabular}




\begin{tabular}{|c|c|c|c|c|c|c|}
\hline \multicolumn{7}{|c|}{ Nacionais } \\
\hline $\begin{array}{c}\text { Silva e } \\
\text { Gonçalve } \\
\text { s-Dias } \\
\end{array}$ & $\begin{array}{c}\text { Mensuração de desempenho } \\
\text { socioambiental: estudo de casos } \\
\text { em negócios sociais brasileiros }\end{array}$ & 2016 & $\begin{array}{l}\text { Revista Pensamento } \\
\text { e Realidade }\end{array}$ & 0 & Empírico & $\begin{array}{l}\text { Google } \\
\text { Scholar }\end{array}$ \\
\hline Coelho & $\begin{array}{l}\text { Indicadores de performance para } \\
\text { projetos sociais: a perspectiva dos } \\
\text { stakeholders }\end{array}$ & 2004 & Revista Alcance & 21 & Empírico & $\begin{array}{l}\text { Google } \\
\text { Scholar }\end{array}$ \\
\hline $\begin{array}{l}\text { Coelho e } \\
\text { Gonçalve } \\
\text { s }\end{array}$ & $\begin{array}{c}\text { Avaliação de projetos sociais: a } \\
\text { perspectiva da comunidade }\end{array}$ & 2011 & Revista Alcance & 11 & Empírico & $\begin{array}{l}\text { Google } \\
\text { Scholar }\end{array}$ \\
\hline Cabral & $\begin{array}{l}\text { Valores e espaço público: } \\
\text { referenciais e instrumentos para a } \\
\text { avaliação de projetos sociais }\end{array}$ & 2011 & $\begin{array}{c}\text { Revista de } \\
\text { Administração } \\
\text { Pública } \\
\end{array}$ & 2 & Empírico & SciELO \\
\hline
\end{tabular}

\section{Figura 1: Trabalhos analisados}

Fonte: dados da revisão

Notou-se dentre os artigos nacionais, que apenas um tratava os termos de empreendedorismo social e mensuração de impacto social, demonstrando assim que a articulação dos termos está mais evidente em trabalhos internacionais. Manetti (2012) nos apresenta que a discussão a respeito de avaliação de impacto socioeconômico acompanha o crescimento da temática de empreendedorismo social, confirmando a relação entre os termos.

Quanto aos trabalhos internacionais analisados pela revisão, foi possível identificar diferentes perspectivas. André, Cho e Laine (2017), apresentaram uma análise sobre a elaboração de avaliação de impacto de um empreendimento social, o qual tinha como missão melhorar a longo-prazo a saúde de uma comunidade oferecendo-lhes água não contaminada. O conhecimento das práticas locais proporcionou o refinamento das operações da organização.

Clark e Brennan (2012) examinaram a questão de como o valor social é criado e medido. Uma perspectiva multidisciplinar de medição de desempenho é apresentada, apoiando-se no argumento de que é necessário estabelecer indicadores. Estes mesmos autores, em outro trabalho, realizaram uma comparação entre casos de quatro organizações, e foi testado um modelo para medição do impacto a longo-prazo. Identificou-se que apenas $37 \%$ das organizações demonstram capacidade de medir indicadores de impacto (Clark \& Brennan, 2016).

Grieco, Michelini e Lasevoli (2014) desenvolveram uma análise hierárquica de clusters com base em uma amostra de 76 modelos de Avaliação de Impacto Social, para agrupá-los em macro categorias e ajudar os empreendedores sociais a escolher o modelo mais adequado às necessidades da sua organização. Hadad e Gauca (2014) elaboraram três princípios que sustentam o modelo de mensuração de impacto, os quais são: sustentabilidade, valor agregado e escalabilidade.

No trabalho de Manneti (2012) é analisado o papel da Blended Value Accounting (BVA) na avaliação do impacto socioeconômico dos empreendimentos sociais, com especial referência ao modelo de mensuração denominado SROI (Retorno Social do Investimento). Moody, Littlepage e Paydar (2015) apresentam a ferramenta SROI, apontando os desafios organizacionais e as lições aprendidas oriundas da implementação da técnica de medição, buscando expandir o conhecimento sobre tais medidas para informar futuros pesquisadores, bem como organizações e financiadores interessados na ferramenta.

Spencer, Brueckner, Wise e Marika (2016) apresentaram uma análise das atividades de uma organização social indígena, a partir de uma estrutura integrada para gerenciamento de desempenho de organizações sem fins lucrativos. A avaliação concentra-se na eficácia social da organização e na sua capacidade de ajudar a gerar renda e emprego aos assistidos.

A partir da revisão realizada, também foram identificados quatro artigos nacionais. O trabalho de Silva e Gonçalves-Dias (2016) caracteriza os métodos empregados de mensuração de impacto social, e também 
identifica as dificuldades encontradas no processo, as motivações do empreendedor para a adoção do método, e os benefícios da mensuração para as organizações.

O artigo de Coelho (2004) apresenta uma metodologia de avaliação para projetos sociais, considerando a perspectiva dos diversos stakeholders envolvidos. Coelho e Gonçalves (2011) contribuem para o processo de avaliação de projetos sociais ao considerarem perspectivas quantitativas e qualitativas, sob o ponto de vista das comunidades envolvidas.

O trabalho de Cabral (2011) apresenta um referencial de avaliação do impacto, dos valores e da economicidade dos benefícios gerados pela gestão social de projetos e iniciativas de organizações sociais locais. Para a autora, a avaliação e as técnicas de mensuração de impacto não estão plenamente presentes no campo das organizações sociais. Entre as razões, encontram-se: falta de profissionalização no setor e o pensamento de que as práticas de avaliação não conferem a existência de uma cultura sistemática e difundida; ausência de uma metodologia específica que apreenda o valor social; insistência em importar técnicas e argumentos tanto da área pública como da área privada.

Essas foram as ideias centrais dos textos que compõe a pesquisa. No próximo tópico serão apresentadas as metodologias e ferramentas de avaliação e mensuração de impacto social, para que possam ser identificadas as metodologias existentes na literatura.

\section{Metodologias e ferramentas de avaliação e mensuração de impacto social}

Existem diferentes formas de avaliar o impacto, e essa diversidade está ligada ao fato de que os empreendimentos variam de tamanho, capacidade, atividades, foco e objetivos, assim, não existe um modelo único adequado para todas as organizações. Portanto, as iniciativas tem o desafio de encontrar uma metodologia apropriada para a sua realidade e para os diferentes interesses de impacto (Grieco, Michelini \& Lasevoli, 2014). Considerando os trabalhos analisados, notam-se diferentes metodologias e ferramentas de mensuração e avaliação, as quais podem ser constatadas na Figura 2.

\begin{tabular}{|c|c|c|}
\hline Foco do artigo & Metodologia e/ou ferramenta & Trabalhos \\
\hline Indicadores & $\begin{array}{lcc}\text { Indicadores } & \text { de desempenho; } \\
\text { Indicadores } & \text { quantitativos e } \\
\text { qualitativos } & & \end{array}$ & $\begin{array}{l}\text { Andre; Cho; Laine (2017); Grieco, Michelini e } \\
\text { Lasevoli (2014); Coelho (2004); Coelho e Gonçalves } \\
\text { (2011) }\end{array}$ \\
\hline Indicadores & $\begin{array}{lll}\text { Metodologia } & \text { Balanced } & \text { Scorecard } \\
\text { adaptada } & & \\
\end{array}$ & Clark e Brennan $(2012 ; 2016)$ \\
\hline $\begin{array}{l}\text { Processo } \\
\text { indicadores }\end{array}$ & Modelo lógico & $\begin{array}{l}\text { Hadad e Gauca (2014); Spencer, Brueckner, Wise e } \\
\text { Marika (2016); Silva e Gonçalves-Dias (2015); Cabral } \\
\text { (2011) }\end{array}$ \\
\hline Indicadores & $\begin{array}{llll}\begin{array}{l}\text { Retorno } \\
\text { (SROI) }\end{array} & \text { Social do } & \text { Investimento } \\
\end{array}$ & Manetti (2012); Moody, Littlepage e Paydar (2015) \\
\hline Indicadores & $\begin{array}{l}\text { Global Impact Investing Rating System } \\
\text { (GIIRS) }\end{array}$ & Silva e Gonçalves-Dias (2015) \\
\hline
\end{tabular}

Figura 2: Metodologias e ferramentas de avaliação e mensuração de impacto social Fonte: as autoras

O trabalho de Andre, Cho e Laine (2017) adotou indicadores sociais de mensuração de cobertura, penetração e regularidade do consumo, os quais possuem metas maleáveis, em comparação aos indicadores de desempenho financeiro. Os indicadores foram desenvolvidos por meio de um projeto de pesquisa-ação e de colaboração ativa entre a organização e os autores do artigo. Eles foram adotados para serem usados regularmente por gerentes e apresentados ao Conselho Administrativo, permitindo o acompanhamento dos resultados e comparações.

Grieco, Michelini e Lasevoli (2014) identificaram e agruparam modelos de mensuração e avaliação de impacto social em quatro clusters: i) modelos baseados em indicadores quantitativos do impacto social 
(por exemplo: número de beneficiários) e do impacto nos empregados (por exemplo: satisfação do empregado e clima organizacional). Esses modelos são fáceis de aplicar (consistindo de não mais de 15 indicadores), são genéricos ou aplicáveis em qualquer setor; ii) modelos caracterizados por variáveis qualitativas e quantitativas. Possui alto grau de complexidade e são aplicados a qualquer setor; iii) modelos que empregam variáveis qualitativas. Devido à sua natureza qualitativa, esses modelos têm um nível de complexidade. Os modelos que pertencem a esse cluster podem ser aplicados a setores específicos e foram desenvolvidos por várias organizações; iv) modelos que usam variáveis qualitativas ou quantitativas e objetivam medir diferentes tipos de impacto. Eles são usados para gerenciamento ou certificação, são aplicados durante o curso das atividades (período de tempo contínuo), e empregados principalmente por empresas e instituições de consultoria.

A metodologia apresentada por Coelho (2004) e Coelho e Gonçalves (2011), também baseada em indicadores, contam com aspectos qualitativos e quantitativos, considerando diferentes stakeholders envolvidos na criação dos indicadores de performance. A metodologia proposta procurou responder questões ligadas a determinadas categorias de análise, as quais envolvem diferentes perspectivas de retorno. Os autores ressaltam que a proposta de avaliação não tem pretensão de oferecer modelos genéricos, prontos para serem aplicados em qualquer realidade. O objetivo foi contribuir com diretrizes e referências para a construção de modelos próprios para cada organização. Enfatiza-se que o avaliador de projetos sociais deve possuir competência técnica e humana, fundamentais para o desenvolvimento de processos participativos.

Clark e Brennan (2012; 2016) apresentaram uma metodologia que é uma adaptação do Balanced Scorecard, de forma que incorporam as perspectivas de curto e longo prazo e o quadro resultante foi denominado de "Matriz de Valor Balanceada", que é uma matriz tridimensional. O modelo tradicional reflete as dimensões do tempo (perspectivas a curto e longo prazo), partes interessadas (perspectivas internas e externas), envolvendo medidas financeiras, satisfação do cliente, processos internos e aprendizado e crescimento. Assim, como terceira dimensão, tem-se o valor social.

Quanto ao modelo lógico, citado por Hadad e Gauca (2014), Spencer, Brueckner, Wise e Marika (2016), Silva e Gonçalves-Dias (2015) e Cabral (2011), é baseado em uma estrutura lógica que apresenta os recursos (entradas), atividades, produtos e serviços (saídas), resultados e impacto social de um projeto. Para construir o modelo lógico, a organização deve iniciar definindo o impacto social que deseja alcançar, para então estabelecer os resultados para atingir o impacto. Depois se define os produtos e serviços a serem desenvolvidos para atingir os resultados, e os recursos a serem empregados.

Quanto ao trabalho de Hadad e Gauca (2014), foi concebido um modelo de medição de impacto social que compreende três categorias de elementos que devem ajudar a medir o impacto social: sustentabilidade, valor agregado e escalabilidade. Em relação à sustentabilidade, a organização deve identificar as fontes para financiar a entidade e suas atividades. Quanto ao valor agregado, deve enfatizar os efeitos psicológicos, sociais, econômicos, ambientais e fatores políticos (caso a atividade principal da empresa se relacione com questões políticas) que a organização tem sobre seu público-alvo. A escalabilidade mede o potencial de expansão da organização, os efeitos, a cobertura da mídia (consciência social) ou mesmo mudanças regulatórias. As autoras defendem que é importante identificar que mudanças precisam ser medidas, no qual os objetivos são transformados em indicadores que devem ser descritos e registrados no início e no final do processo. Valores, visão, missão, metas e objetivos tem que ser definidos, pois as atividades serão guiadas por esses cinco elementos.

A abordagem apresentada por Spencer, Brueckner, Wise e Marika (2016) envolve determinadas dimensões de análise, quais sejam: i) insumos, que são os recursos necessários, ii) capacidade organizacional, que são as características humanas e estruturais da organização, iii) saídas, que são os produtos e serviços desenvolvidos, iv) valor público, que é a criação de valor em termos de bem-estar e felicidade, capital social ou inclusão social, e v) rede e legitimidade institucional, que envolve a gestão de 
relacionamentos com as partes interessadas da organização e sua capacidade de desenvolver e manter parcerias.

Silva e Gonçalves-Dias (2015) apresentam que na mensuração de desempenho socioambiental os elementos da cadeira de geração de valor social (modelo lógico) devem ser considerados. Para que as empresas possam comparar o seu desempenho social e ambiental e comunicá-lo aos diversos atores que compõem o campo dos negócios sociais, são apresentadas alternativas que utilizam de uma linguagem comum (indicadores e sistemas para calcular impacto). Os empreendimentos estudados pelos autores utilizam um sistema de mensuração de desempenho socioambiental, o GIIRS (Global Impact Investing Rating System), que produz uma avaliação considerando como resultado uma pontuação do impacto positivo gerado pela organização avaliada. Como é possível perceber, este trabalho encontra-se presente em duas categorias na Figura 2, por abordar o modelo lógico e o sistema GIIRS.

Cabral (2011) construiu um Mapa de Bens Públicos, que é um instrumento para verificar o cumprimento da missão da organização, de forma que vincula valores e resultados como benefícios mensurados por indicadores. O Mapa pode ser tratado como um desenho do projeto, no qual são apresentadas as conexões entre os elementos: recursos, atividades, produtos, resultados e benefícios, de modo a garantir a articulação entre eles a fim de cumprir os objetivos almejados. Quanto à operacionalização, é necessário que envolva um grupo de responsáveis de diferentes áreas organizacionais e os gestores do projeto reunidos em um ambiente dinâmico e dialogado. Haverá o preenchimento das lacunas a serem respondidas, seguindo a ordem: benefício, bens públicos, resultados, recursos e atividades. Ou seja, a primeira pergunta se refere aos benefícios pretendidos com o projeto, como os valores fundamentais assumidos pelo empreendimento, que envolve a questão social abordada.

Manetti (2012) e Woody, Littlepage e Paydar (2015), abordaram o uso do SROI (Retorno Social do Investimento), o qual tem como objetivo medir o valor econômico e social gerado por uma organização em uma comunidade local onde opera para obter uma quantificação do impacto social gerado. O resultado final do processo de implementação da análise SROI é um indicador que representa o retorno em termos socioeconômicos para cada unidade monetária gasta no projeto e/ou na organização como um todo. O retorno social do investimento usa elementos de análise de custo-benefício, os quais são quantificados e comparados para avaliar a conveniência de uma intervenção expressa em unidades monetárias. A partir desta ferramenta, nota-se o risco da financeirização dos instrumentos de mensuração do impacto das organizações sociais.

De acordo com Manetti (2012), o SROI é um instrumento projetado para monetizar o impacto em uma lógica quase comercial. Para o empreendimento o mais importante é compreender o processo de criação de valor socioeconômico, independentemente de sua monetização. Deve-se, portanto, entender a mensuração de impacto além da elaboração de indicadores, mas compreendendo também a discussão a respeito das etapas a serem seguidas para que o impacto seja alcançado e as transformações sociais sejam realizadas. Essa questão será discutida no próximo tópico.

\section{Mensuração e avaliação de impacto social: delineando o processo}

Dentre as metodologias e ferramentas identificadas nos artigos pesquisados, algumas tratavam de mensuração de impacto social envolvendo todo o processo na geração de valor social, destacando os elementos a serem trabalhados (recursos, atividades, resultados etc.), e as relações causais entre eles, ou seja, as relações entre os elementos. Outras tratavam apenas do desenvolvimento de indicadores para apresentar o resultado atingido, sem discutir as variáveis envolvidas no processo, desta forma focando apenas na medição.

Observou-se que $75 \%$ dos trabalhos teve foco apenas na medição, enquanto $25 \%$ tratavam do processo em conjunto com a medição. A partir disso, nota-se a importância de discutir a mensuração e avaliação 
de impacto social em uma perspectiva que envolva todos os mecanismos que levarão às mudanças sociais almejadas pelo projeto, como defendido por Rogers e Weiss (2007).

Para Hadad e Gauca (2014) os métodos presentes na literatura para medir impacto social tendem a quantificar tudo em dinheiro, além de negligenciar a importância do impacto psicológico que uma empresa pode ter sobre seu público-alvo. O impacto é medido na perspectiva das empresas e dos investidores, negligenciando a participação dos beneficiários, os quais são alvo do projeto. Por fim, discute-se que não há legislação adequada para regular este campo de mensuração de impacto, e nenhum órgão de controle especializado para supervisionar a atividade com essas iniciativas.

Coelho (2004) defende a necessidade de estudos sobre avaliação de projetos sociais, e relaciona a isso a curta duração de vida de diversas ações sociais. A autora apresenta que a falta de comprometimento com resultados, por questões motivacionais altruístas, ou por questões motivacionais institucionais através do marketing social (impacto social pouco significativo supervalorizado pela estratégia de comunicação), são exemplos práticos.

O processo de avaliação de impacto social deve envolver não apenas a utilização eficiente dos recursos, mas buscar identificar se as ações contribuíram de fato para traduzir o impacto social para todos os envolvidos no processo (Coelho, 2004). Para Coelho (2004) nem sempre é possível uma vinculação direta entre o programa e os resultados atingidos, porque podem existir outros fatores que exercem influência sobre o contexto. Outra questão que pode ocorrer, é que o empreendedor pode ser muito eficaz em prestar os serviços, mas os beneficiários podem não perceber o benefício esperado, correndo o risco do impacto a longo-prazo não ser alcançado (Clark \& Brennan, 2012).

De acordo com Hadad e Gauca (2014), uma abordagem para medir impacto que não inclua a transferência de poder para as partes interessadas é apenas marketing. Para as autoras, os problemas sociais são difíceis de definir, e para tanto, é preciso compreender as necessidades da sociedade, por meio de um trabalho que demanda interesse, atenção, tempo e empatia. Cada empreendimento e inovação social visam objetivos diferentes, com base no contexto cultural, na compreensão do problema social a ser abordado, no valor a ser criado, e nas questões éticas envolvidas (Hadad \& Gauca, 2014).

A aproximação com a comunidade e o entendimento do contexto são questões que precisam ser discutidas na maioria dos textos, por isso há de ser destacado o trabalho de Andre, Cho e Laine (2017), no qual os autores relataram um caso de uma organização que percebeu que a melhoria do impacto social não aconteceria por meio de melhorias técnicas, o que demandou um estudo de abordagem antropológica para entender a cultura local e suas práticas relacionadas ao uso da água. Nesse trabalho a vOz dos beneficiários passou a ser ouvida e a estratégia da organização mudou, passando a ter um amplo conhecimento sociocultural do contexto. Destaca-se a necessidade de entender a realidade local, a partir do momento que identificou erro nos pressupostos da organização e a falta de conhecimento do seu público-alvo.

Portanto, são questões primordiais para se discutir ao apresentar abordagens para mensuração de impacto social, ou para desenvolver um projeto de impacto ou um empreendimento social. A partir da análise das diferentes metodologias, entende-se que o processo de mensuração e avaliação de impacto social tem como base um modelo lógico, geralmente baseado nos seguintes elementos: recursos, atividades, produtos/serviços, resultados e impactos (Andre, Cho \& Laine, 2017; Cabral, 2011; Silva \& GonçalvesDias, 2015; Spencer et al., 2016), apresentando o vínculo entre a missão e as ações realizadas (Cabral, 2011).

Essa atividade envolve a participação de diferentes atores, onde a comunidade, a qual contempla os beneficiários que serão impactados pelo projeto, devem ser o centro da análise. A mensuração de impacto precisa envolver constantemente premissas por trás de todo o processo, de forma que não mantenha o foco apenas no resultado final (Rogers \& Weiss, 2007). É importante entender as relações entre os 
elementos do modelo lógico, para que os mesmos não sejam interpretados de forma linear (sem apresentar relações). Sendo assim, os pressupostos precisam ser constantemente avaliados, conforme apresentado por André, Cho e Laine (2017).

Portanto, para desenvolver um projeto social, inicialmente deve-se identificar o impacto a ser alcançado. Para isso é necessário o contato com a realidade local a fim de buscar conhecimento sobre o contexto, a história e a cultura da comunidade, e dessa forma entender quais são os problemas sociais que existem e quais são as necessidades que devem ser atendidas. A partir disso é possível identificar soluções inovadoras para os problemas sociais e ambientais, e estabelecer os objetivos a serem atendidos, por meio de um trabalho em conjunto com as partes interessadas. Diante disso, o processo começa a ser estruturado, e a estratégia de impacto a ser trabalhada.

Para se atingir os objetivos de impacto social, deve-se estabelecer quais resultados, produtos/serviços, atividades e recursos serão necessários. Por fim, é fundamental desenvolver indicadores para acompanhar esses elementos, e assim avaliar os resultados atingidos. Caso sejam identificados erros, ou caso a solução desenvolvida não faça sentido para os beneficiários, os pressupostos por trás das atividades deverão ser revistos e um novo planejamento deverá ser elaborado.

Nota-se assim, como defendido por Coelho e Gonçalves (2011), que o avaliador de projetos sociais deve primar pela capacidade de unir competência técnica e humana, para uma melhor gestão de processos participativos. André, Cho e Laine (2017) também destacam a importância de trabalhar aspectos técnicos e sociais, para envolver toda a complexidade do processo. E por ser complexo, pode contar com o apoio de consultorias (Andre, Cho \& Laine, 2017; Moody, Littlepage \& Paydar, 2015; Silva \& Gonçalves-Dias, 2015), ou até mesmo de universidades, as quais podem ser estratégicas nesse processo, por meio da geração de conhecimento científico para subsidiar a mensuração e ao mesmo tempo possibilitar integração e diálogo com a sociedade (Silva \& Gonçalves-Dias, 2016). Envolver pessoas capacitadas para estruturar uma metodologia que envolva a participação de todas as partes interessadas e que atenda ao objetivo de impacto social é sempre algo a ser considerado.

\section{Considerações finais}

O objetivo do trabalho foi identificar e discutir as metodologias e ferramentas para acompanhar, mensurar e avaliar o impacto social por empreendimentos sociais. Assim, foram analisados 12 artigos, a partir de duas categorias, as quais são: "Metodologias e ferramentas de avaliação e mensuração de impacto social" e "Foco do artigo (processo, indicadores de resultado ou ambos)". Após a análise foi compreendido o processo de geração, de acompanhamento e de mensuração do impacto social proporcionado pelas organizações sociais, a fim de contribuir para a discussão da temática.

Foram encontrados poucos trabalhos internacionais e nacionais que tratam deste assunto, confirmando o que foi apresentado por Nicholls (2009) e So e Staskevicius (2015), de que esse campo ainda não está bem estruturado, faltando mais estudos e pesquisas a respeito da temática de mensuração e avaliação de impacto de empreendimentos sociais.

Foi possível identificar que existem diferentes formas de avaliar o impacto social, e a escolha de metodologias e ferramentas específicas está relacionada à realidade da organização. Diante dessa variedade se destaca a elaboração de dimensões de análise e indicadores de desempenho, e a escolha de metodologias e ferramentas reconhecidas, como o Retorno Social do Investimento (SROI), o Global Impact Investing Rating System (GIIRS) e o modelo lógico, os quais, de modo geral, buscam apresentar os resultados de impacto obtidos pela organização e auxiliam na identificação de formas de melhorar o desempenho e evidenciar o valor social alcançado. 
Assim, o trabalho contribui em oferecer um novo olhar a respeito da mensuração de impacto, devido ao fato de que é predominante o uso de metodologias com o foco voltado apenas na elaboração de indicadores, sem discutir o processo por trás da estratégia de gerar impacto social. Identificaram-se também casos onde a mensuração é gerada apenas para atender aos interesses externos de investimento, sendo que deveria partir da estratégia da organização ao definir sua missão social, para atingir um impacto significativo para a comunidade, pois é a partir dela que desenvolve o projeto.

Nota-se que há a discussão da avaliação e mensuração de impacto social na perspectiva de uma estrutura lógica, onde se apresentam estabelecidos de forma linear os recursos, as atividades, os produtos e os resultados que serão alcançados, mas detectou-se uma carência de uma discussão que envolva os processos de cada uma das etapas para alcançar um resultado efetivo e gerar as mudanças sociais pretendidas. Além disso, notou-se também a falta de abordagens que destaquem a participação dos beneficiários e da comunidade nesse processo, a fim de enfatizar a importância de uma aproximação entre os envolvidos para o desenvolvimento de um projeto social.

O trabalho evidencia a necessidade de ao se desenvolver um projeto que almeja gerar impacto social, na perspectiva de um impacto positivo, que primeiramente haja um planejamento que pressuponha uma imersão na realidade da comunidade que se pretende atuar, para entender suas necessidades e as especificidades locais. Para que a partir desse momento, de forma dialógica, seja desenvolvido um projeto com foco em mudanças necessárias. O impacto social irá ocorrer na medida em que estiver alinhado com as necessidades locais, não se pautando apenas em análises objetivas e quantitativas, mas também considerando aspectos subjetivos, os quais devem estar apoiados em indicadores qualitativos, a fim de apresentar os problemas existentes e os pressupostos a serem alterados para atingir o impacto almejado.

Como sugestões de pesquisas futuras, sugere-se realizar um mapeamento a respeito do uso por empreendimentos sociais de metodologias de mensuração e avaliação de impacto social, para analisar como elas se caracterizam e em que contribuem para a melhoria dos processos a fim de gerar impacto positivo para as comunidades.

\section{Referências}

Abdo, D., \& Paris, C. M. (2017). Social entrepreneurship in the UAE: challenges and recommendations. Int. J. Sustainable Society, v. 9, n. 1.

Alvord, S. H., Brown, L. D., \& Letts, C. W. (2004). Social Entrepreneurship and societal transformation. An exploratory study. The Journal of Applied Behavioral Science, v.40, n.3.

Andre, K., Cho, C. H., \& Laine, M. (2017). Reference points for measuring social performance: Case study of a social business venture. Journal of Business Venturing.

Ashoka Empreendedores Sociais \& McKinsey\&Company. (2001). Empreendedores Sociais Sustentáveis: Como elaborar planos de negócio para organizações sociais. São Paulo: Peirópolis.

Austin, J., Stevenson, H., \& Wei-Skillern, J. (2006). Social and Commercial Entrepreneurship: Same, Different and Bolth? Entrepreneurship: Theory and Practice.

Azevedo, L. P. (2015). As distintas percepções sobre o empreendedorismo social. Dissertação (Mestrado em Gestão Empresarial) - Escola Brasileira de Administração Pública e de Empresas da Fundação Getúlio Vargas, São Paulo.

Bacq,S., \& Janssen, F. (2011). The multiple faces of social entrepreneurship: A review of definitional issues based on geographical and thematic criteria. Entrepreneurship and Regional Development: An International Journal, n. 23, n. 5-6, p. 373-403.

Bardin, L. (2009). Análise de Conteńdo. Lisboa, Portugal, Edições 70, LDA. 
Barki, E., Comini, G., Cunlife, A., Hart, S., \& Rai, S. (2015). Social Entrepreneurship and Social Business: Retrospective and Prospective Research. Revista de Administração de Empresas-FGV. São Paulo, V.55 n. 04, jul/ago.

Barman, E. (2007). What is the bottom line for nonprofi organizations? A history of measurement in the British Voluntary Sector. Voluntas.

Botelho, L. L. R., Cunha, C. C. A., Macedo, M. (2011). O método da revisão integrativa nos estudos organizacionais. Revista Eletrônica Gestão e Sociedade. Belo Horizonte, v. 4, n. 11, p. 121-136.

Brandão, D., Cruz, C., \& Arida, A.L. (2014). Métricas em Negócios de Impacto Social: Fundamentos. São Paulo: ICE/MOVE, 15p.

Cabral, E. H. S. (2011). Valores e espaço público: Referenciais e instrumentos para a avaliação de projetos sociais. Revista de Administração Pública - RAP, v. 45, n. 6.

Certo, S. T., \& Miller, T. (2008). Social entrepreneurship: key issues and concepts. Business Horizons, v. 51, p. 267-271.

Choi, N., \& Majumdar, S. (2013). Social entrepreneurship as an essentially contested concept: opening a new avenue for systematic future research. Journal of Business Venturing.

Church, C., \& Rogers, M. (2006). Designing for Results: Integrating Monitoring and Evaluation in Conflict Transformation Programs. Washington, DC: Search for Common Ground.

Clark, C., \& Brennan, L. (2012). Entrepreneurship with social value: A conceptual model for performance measurement. Academy of Entrepreneurship Journal, v. 18, n. 2.

. (2016). Social entrepreneurship: A global model for evaluating long-term impact. International Journal of Entrepreneurship, v. 20, n. 1.

Clifford, J., Markey, K., \& Malpani, N. (2013). Measuring Social Impact in Social Enterprise: The state of thought and practice in the UK. London. E3M.

Coelho, M. Q. (2004). Indicadores de performance para projetos sociais: A perspectiva dos stakeholders. Revista Alcance, v. 11, n. 3., p. 423-444.

Coelho, M. Q., \& Gonçalves, C. A. (2011). Avaliação de projetos sociais: A perspectiva da comunidade. Revista Alcance, v. 18, n. 4, p. 436-447.

Corner, P. D., \& Ho, M. (2010). How opportunities develop in social entrepreneurship. Entrepreneurship: Theory and Practice.

Dacin, M. T., Dacin, P. A., \& Tracey, P. (2011). Social entrepreneurship: A critique and future directions. Organization Science, v. 22, n. 5, p.1203-1213.

Dees, J. G. (1998). The meaning of social entrepreneurship. Boston, MA: Harvard Business School.

Defourny, J., \& Nyssens, M. (2010). Conceptions of social enterprise and social entrepreneurship in Europe and the United States: Convergences and divergences. Journal of Social Entrepreneurship, v. 1, n. 1, p. 32-53.

Grieco, C., Michelini, L., \& Lasevoli, G. (2014). Measuring value creation in social enterprises: A cluster analysis of social impact assessment models. Nonprofit and Voluntary Sector Quarterly.

Hadad, S., \& Gauca, O. (2014). Social impact measurement in social entrepreneurial organizations. Management \& Marketing. Challenges for the knowledge society, v. 9, n. 2, p. 119-136.

Kuyumjian, R., Souza, E. M., \& Sant'anna, S. R. (2014). Uma análise a respeito do desenvolvimento local: o empreendedorismo social no Morro do Jaburu - Vitória (ES), Brasil. Rev. Adm. Pública, v. 48, n. 6. 
Mair, J., \& Marti, I. (2006). Social entrepreneurship research: A source of explanation, prediction, and delight. Journal of World Business, v. 41, p. 36-44.

Manetti, G. (2012). The role of blended value accounting in the evaluation of socio-economic impact of social enterprises. Voluntas.

Moody, M., Littlepage, L., \& Paydar, N. (2015). Measuring Social Return on Investment: Lessons from organizational implementation of SROI in the Netherlands and The United States. Nonprofit Management \& Leadership, v. 26, n. 1.

National Council for Voluntary Organizations. (2013). Inspiring Impact: The Code of Good Impact Practice.

Neck, H., Brush, C., Allen, E. (2009). The landscape of social entrepreneurship. Business Horizons, v. 52, p. 13-19.

Nicholls, A. (2009). "We do good things, don't we?": "Blended Value Accounting" in social entrepreneurship. Accounting, Organizations and Society, v. 34, p. 755-769.

Ogain, N. E. (2015). Impact Measurement in Impact Investing: Learning from Practice. NESTA Impact Investments.

Oliveira, E. M. (2004). Empreendedorismo social no Brasil: atual configuração, perspectivas e desafios. Revista da FAE, Curitiba, v.7, n.2, p.9-18, jul./dez.

Parkinson, D., \& Wadia, A. (2008). Keeping on Track: A guide to setting and using indicators. Performance Hub.

Rogers, P. J., \& Weiss, C. H. (2007). Theory-based evaluation: reflections ten years on. Theory-based evaluation: past, present and future. New Directions for Evaluation.

Saebi, T., Foss, N. J., \& Linder, S. (2018). Social Entrepreneurship Research: Past Achievements and Future Promises. Journal of Management.

Santos, F. M. (2012). A positive theory of social entrepreneurship. Journal of Business Ethics, p. 335-351.

Seelos, C., \& Mair, J. (2005). Social entrepreneurship: Creating new business models to serve the poor. Business Horizons, v. 48, p. 241-246.

Silva, A. P. A., \& Gonçalves-Dias, S. L. F. (2015). Mensuração de desempenho socioambiental: Estudo de casos em negócios sociais brasileiros. Revista Pensamento e Realidade, v. 30, n. 2.

So, I., \& Staskevicius, A. (2015). Measuring the "Impact" in Impact Investing. Harvard Business School.

Spencer, R., Brueckner, M., Wise, G., \& Marika, B. (2016). Australian indigenous social enterprise: measuring performance. Journal of Enterprising Communities: People and Places in the Global Economy, v. 10, n. 4.

Tyszler, M. (2007). Mudança social: uma arte? Empreendimentos sociais que utilizam a arte como forma de mudança. RAP, v. 41, n. 6.

Weiss, C.H. (1995). Nothing as Practical as Good Theory: Exploring Theory Based Evaluation for Comprehensive Community Initiatives for Children and Families. In: Connel, J., Kubisch, A., Schorr, L., \& Weiss, C. H. New Approaches to Evaluating Community Initiatives: Concepts, Methods and Contexts. Washington, D.C.: The Aspen Institute.

Yunus, M., Moingeon, B., \& Lehmann-Ortega, L. (2010). Building Social Business Models: Lessons from the Grameen Experience. Long Range Planning, vol 43. 